\section{Rejilla de Amsler versus optotipo de visión próxima y de lectura: autodiagnóstico precoz de la DMAE neovascular}

\section{Amsler grid versus near acuity and reading vision chart: early self-diagnosis in neovascular AMD}

\section{Sr. Director:}

Hemos leído con interés y felicitamos a los Dres. Flores-Moreno y cols. sobre «Tratamiento de la DMAE: una asignatura pendiente». Creemos es importante el tema del coste del tratamiento pero no la única asignatura pendiente, dado que dichos tratamientos exitosos están obligándonos a mantener de manera continuada durante años y «sine die» las reinyecciones para evitar las constantes recidivas.
Como detectar estas recidivas precozmente está constituyendo un verdadero quebradero de cabeza para los clínicos.

Basándonos en el hecho observacional cotidiano de que uno de los principales motivos de pérdida de calidad visual de los pacientes con DMAE es no poder leer, el objetivo del presente estudio fue investigar la eficacia de asociar un Optotipo de Visión Próxima y Lectura a la clásica Rejilla de Amsler en el control doméstico periódico y diagnóstico precoz de la Degeneración macular asociada a la edad (DMAE) neovascular o húmeda.

La lámina diseñada para el estudio presenta por un lado las diferentes versiones de la rejilla de Amsler y en el contrario la escala de optotipos de cerca para lectura, con variante numérica y de círculos con apertura orientada para ser utilizable en situaciones culturalmente complejas o límite (fig. 1). Los pacientes eran instruidos a usarla semanalmente. Además de realizar el control clásico de la reji-

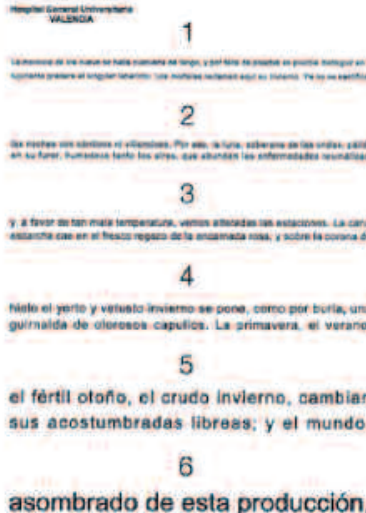
no distingue tal de cual. $Y$ la 7

progenie misma de estos males proviene de nues8

tras querellas y disensiones. ¡Nosotros so9 mos sus padres y 10 engendradores! 9536704128
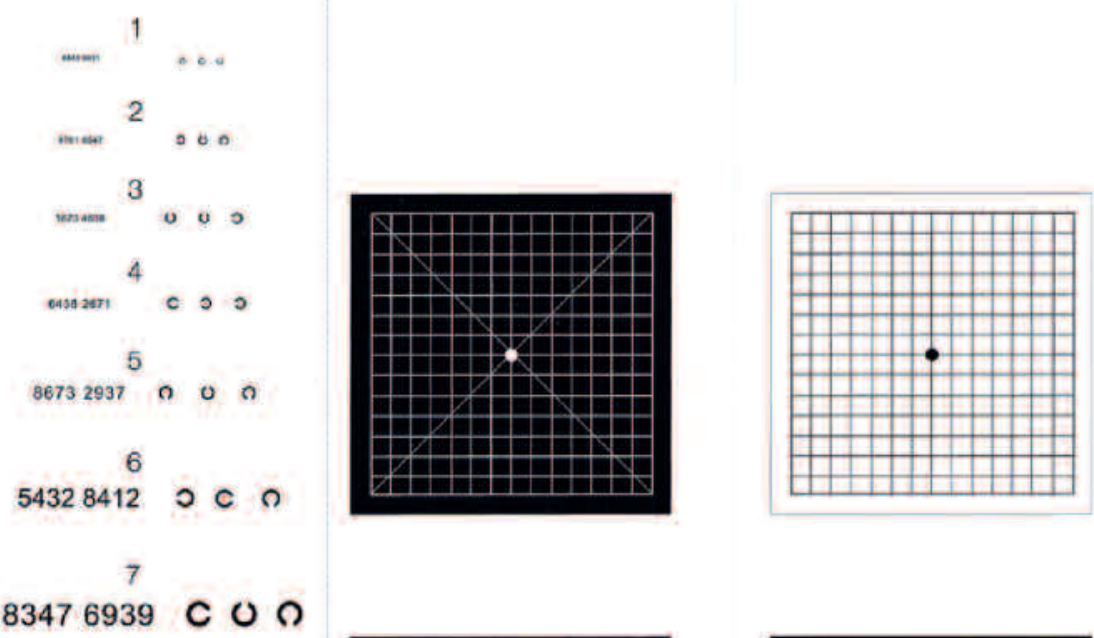

68132479 ○ C O
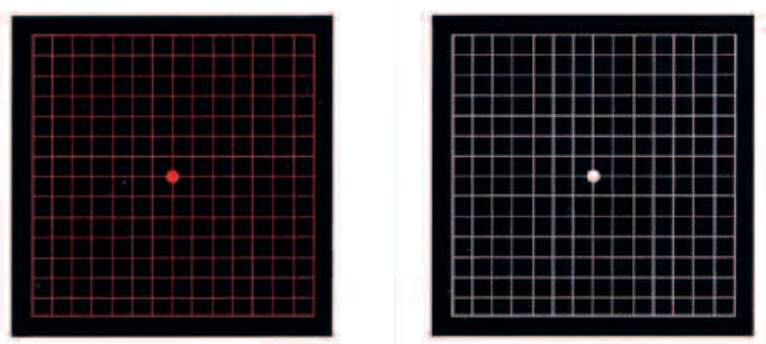

Fig. 1: Test doméstico «doble» con Rejilla de Amsler y Optotipo de visión de cerca para el diagnóstico precoz de la DMAE neovascular (cortesía de Laboratorio Pharma-Lepori). 
lla (siempre con idéntica gafa de cerca, luz y distancia), debían establecer la línea más pequeña capaz de leer individualmente en cada ojo y marcarla, acudiendo de urgencia en caso de que cualquiera de los dos tests se alterara: variaciones en la rejilla (aumento o aparición «de novo» de escotoma o de metamorfopsia), o aumento en la dificultad de lectura de la línea más pequeña previamente leíble.

De manera prospectiva se estudiaron un total de 20 pacientes de alto riesgo de membrana neovascular (ojo único con drusas blandas) o pacientes con DMAE húmeda ya tratada e inactivada con diferentes pautas de tratamiento (intravítreas de bevacizumab, ranicizumab o triamcinolona, terapia fotodinámica) pero con riesgo de recidivas de su membrana neovascular, que habían previamente sido instruidos a realizar el doble control de rejilla y de la línea máxima de lectura capaces de leer, y que acudieron por propia iniciativa al aparecer «de novo» una membrana neovascular $(\mathrm{n}=6)$ o reactivarse la previamente inactiva $(n=14)$, confirmándose en todos los casos dicha actividad por OCT y angiografía. En el $70 \%$ de los casos $(n=14)$ el único signo de alarma era la disminución en la escala de lectura, en un $25 \%(n=4)$ el paciente percibía la alteración en ambos test (rejilla de Amsler y optotipos de cerca), y sólo en un $5 \%(n=2)$ el único signo alterado era la rejilla. El $100 \%$ de los casos con visiones peores (AV de lejos igual o peor de 3/10) y el $100 \%$ de los casos de DMAEs neovasculares ya previamente tratadas, el único signo alterado era la disminución de la capacidad de lectura y en ningún caso la modificación de la rejilla de Amsler. Esto demuestra la mayor sensibilidad en el diagnóstico precoz del test de lectura frente al Amsler.

Estudios previos han demostrado que a pesar de ser la rejilla el test de autodiagnóstico universalmente establecido desde su descripción por el suizo Amsler en 1953 hasta nuestros días (1), su eficacia máxima o sensibilidad en el diagnóstico precoz de la DMAE varía entre un $40 \%$ y menos del $10 \%$ dependiendo del grado de instrucción sobre su uso y nivel intelectual de los pacientes $(2,3)$. Además de la falta de cumplimiento doméstico y el no ser interactivo con la consecuente falta de control de calidad del método de realización, las causas fisiopatológicas de tan baja rentabilidad en el diagnóstico precoz de la rejilla son: A) Fenómeno cerebral de «rellenado» (Filling-in phenomenon), o capacidad cerebral de ignorar el escotoma (fig. 2). B) Incapacidad de detectar escotomas pequeños menores de 6

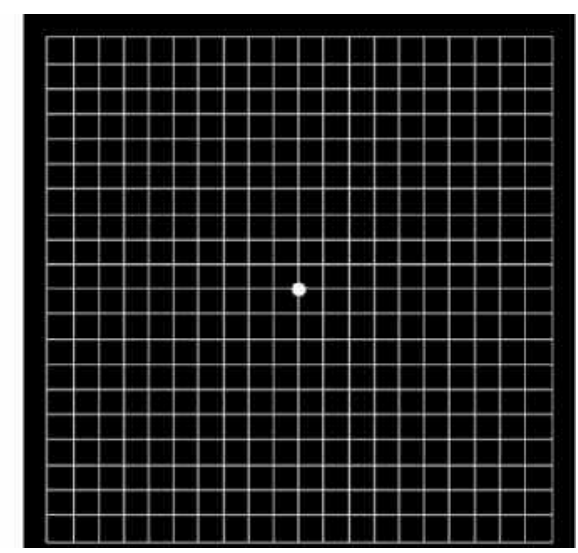

Fig. 2: Demostración del «fenómeno de rellenado» (Filling-in phenomenon). Con el ojo izquierdo cerrado, fijar la cruz gris de la izquierda mientras observamos la rejilla de Amsler a unos $25 \mathrm{~cm}$. No queda ningún hueco o escotoma perceptible entre las líneas, a pesar de la proyección del punto ciego fisiológico sobre la rejilla.

grados. C) Incapacidad de mantener la fijación central mientras se mantiene la atención en la periferia, con «fenómeno de agrupamiento» (Crowding phenomenon) de las líneas periféricas. Dicha sensibilidad es mayor en la rejilla de fondo negro que en la de fondo blanco (4) y podría incrementarse hasta 5 veces incorporando filtros polarizados cruzados en montura de pruebas para visualizar la rejilla $(5,6), \mathrm{o}$ también utilizando el nuevo Preferential Hiperacuity Perimeter (PHP, Zeiss) hasta un $75 \%$, pero su elevado coste, obligatoriedad de uso hospitalario y falsos positivos de más del $15 \%$, no permiten considerarlo una alternativa válida (7). El Macular Mapping Test, test con posibilidades de uso doméstico al ser libremente accesible por internet, precisa la colaboración de un familiar entrenado y con buena agudeza visual, una inversión de tiempo y grado de intelectualidad considerable lo que lo dificulta para poderlo universalizar como autotest de diagnóstico precoz o «screening» $(8,9)$.

La utilidad de incorporar el optotipo de visión cercana en la monitorización de la progresión de la patología macular podría posiblemente demostrar su utilidad en la monitorización doméstica de otras patologías maculares frecuentes y graves, como el edema macular diabético y de otras etiologías (uveítis, etc.), donde no está establecido ningún test como estandarizado para el diagnóstico precoz de su progresión (10). Dado el explosivo aumento de casos de DMAE con el progresivo envejecimiento de la pirámide poblacional, la dificultad para diagnosticar 
precozmente las recidivas postratamiento, su fácil realización y gran sensibilidad, creemos que la monitorización del optotipo de visión próxima debería ser incorporado como un test más entre las medidas generales de salud pública que se realizan de manera rutinaria en toda la población a partir de la edad de 65 años, aun sin factores oftalmológicos de riesgo inmediato, pero especialmente si estos existen (ojo único por DMAE contralateral o drusas blandas).

Concluimos que es mucho más sensible para el diagnóstico precoz asociar ambas pruebas (Optotipo de Cerca/Lectura y Rejilla de Amsler), tanto en la monitorización post-tratamiento como en el diagnóstico precoz de los pacientes con riesgo de DMAE neovascular (drusas, DMAE en el ojo contralateral, etc), que sólo utilizan la Rejilla. Utilizar el optotipo de cerca no supone un consumo de tiempo extra, es de más fácil comprensión y realización por los pacientes de edad avanzada que la propia rejilla, les obliga al uso de la preceptiva graduación de cerca que tan habitualmente olvidan al realizar sólo la rejilla disminuyendo todavía más su ya escasa sensibilidad, permite ser utilizado con las ayudas visuales especiales de baja visión habituales del paciente (lupas, etc.), y no requiere instrucciones complementarias. La monitorización de la DMAE neovascular obliga a incorporar el optotipo de visión próxima de lectura por su alta sensibilidad, muy superior a la clásica rejilla de Amsler.

Díaz-Llopis M, Cervera E, García-Delpech S, Udaondo P, Salom D, Montero J

Facultad de Medicina. Universidad de Valencia.
Hospitales Universitarios

La Fe y General de Valencia.

Universidad Católica San Vicente Mártir

E-mail: manuel.diaz@uv.es

\section{BIBLIOGRAFÍA}

1. Amsler M. Earliest symptoms of diseases of the macula. $\mathrm{Br}$ J Ophthalmol 1953; 37: 521-537.

2. Shuchard RA. Validity and interpretation of amsler grid reports. Arch Ophthalmol 1993; 111: 776-780.

3. Fine AM, Elman MJ, Ebert EM, Prestia PA, Starr JS, Fine $S L$. Earliest symptoms caused by neovascular membranes in the macula. Arch Ophthalmol 1986; 104: 513-514.

4. Augustin AJ, Offerman I, Lutz J, Schmidt-Erfurth U, Tornambe $P$. Comparison of the original amsler grid with the modified amsler grid: results for patients with age-related macular degeneration. Retina 2005; 25: 443-445.

5. Wolfe KA, Sadun AA.Threshold Amsler grid testing in diabetic retinopathy. Graefes Arch Clin Exp Ophthalmol 1991; 229: 219-223.

6. Wall M, Sadum AA. Threshold Amsler grid testsing. Cross-polarizing lenses enhance yield. Arch Ophthalmol 1986; 104: 520-523.

7. Alster Y, Bressler NM, Bressler SB, Brimacombe JA, Crompton RM, Duh YJ, et al. Preferential Hyperacuity Perimetry (PreView PHP) for detecting choroidal neovascularization study. Ophthalmology 2005; 112: 1758-1765.

8. Bartlett H, Davies LN, Eperjesi F. The macular mapping test: a reliability study. BMC Ophthalmology 2005; 5: 18.

9. Trauzettel-Klonsinski S, Biermann P, Hahn G, Weisman M. Assessment of parafoveal finction in maculopathy: a comparison between the Macular Mapping Test and kinetic Manual Perimetry. Graefes Arch Clin Exp Ophthalmol 2003; 241: 988-995.

10. Kiss CG, Barisani-Asenbauer T, Maca S, RitcherMueksch S, Radner W. Reading performance of patients with uveitis-associated cystoid macular edema. Am J Ophthalmol 2006; 142: 620-624. 